\title{
In Ovo Route of Administration
}

National Cancer Institute

\section{Source}

National Cancer Institute. In Ovo Route of Administration. NCI Thesaurus. Code C149575.

Administration of a veterinary medicinal product through the shell into an embryonated egg. 